\title{
Dynamical model of a new type of self-balancing tractor-trailer-bicycle
}

\author{
Wei Zhuang ${ }^{1}$, Gaoming $\mathrm{Li}^{1}$, Ruixin Zhang ${ }^{1}$, Xiao $\mathrm{Su}^{1}$, and Yonghua Huang ${ }^{1, *}$ \\ ${ }^{1}$ School of Mechanical and Electrical Engineering, Guilin University of Electronic Technology, \\ Guilin, China, 541004
}

Keywords: Self-balancing tractor-trailer-bicycle, Dynamical model, Chaplygin equation.

\begin{abstract}
In this paper, we focused on a Self-Balancing Tractor-Trailer-Bicycle(TTB) and developed an under-actuated dynamical model for the system. The bicycle is characterized with two parts, that is a tractor and a trailer, and considering the nonholonomic constrains from no-slipping contacts of its three wheels and the flat ground, we presented a dynamical model for the bicycle by using Chaplygin equation. The model suggest that the TTB should be an under-actuated system with three DOF (degree of freedom) and there are two driving-torque inputs. An inverse dynamics and a virtual prototype simulations are given to demonstrate the correctness of the proposed dynamical model.
\end{abstract}

\section{Introduction}

Self-balancing bicycle is the combination of bicycle mechanism and balance control technology. For this kind of two-wheeled mechanism, on the one hand, it can satisfy ones' needs of convenient travelling and labor-saving due to its lightweight and flexible body; on the other hand, the bicycle riders hope in some case that it can balance automatically, which can get rid of the dependence on ones' "driving". So far, the research on the self-balancing bicycle can be grouped into two types: "without mechanical regulator" and "with mechanical regulator".

Researchers who focus on self-balancing bicycle without mechanical regulators include Jones[1], Tanaka[2], Kooijman[3], Huang[4,5], and Li[6], etc. These researchers believed that the unmanned bicycle can achieve the dynamic balance of the body by governing the handlebar turning and the wheels running without adding additional mechanical regulators.

In the literatures [4-5], Huang introduced the principle of instantaneous rotation axis to analyze the constraints of bicycle robots, and used Lagrange method to establish its dynamic model. Huang also designed a motion controller based on partial feedback linearization method, and finally gave some physical prototype experiments, e.g., in situ, circular motion, linear balanced walking, etc.

Researchers who study self-balancing bicycles with mechanical regulators include Lee[7], Bui[8], Liu[9], Jin[10], Yin[11], Kim[12], etc. These researchers designed

\footnotetext{
*Corresponding author: huangyonghuaxj@sina.com
} 
mechanical adjusting devices of moving or rotating for their bicycles. They stressed the dominant role of the mechanical adjusting devices in maintaining the balance of the body. The balance adjustment mechanism includes inertial flywheel (or rotating rod, or pendulum rod), translation mass block, mechanical gyro, etc.

At present, the research of self-balancing bicycle is mostly focused on the self-balancing of the body, and few of them can pay attention to the problem of the payload capacity of the system. Since the self-balancing bicycle without mechanical regulator has a simpler structure, fewer driving motors, lighter weight, and of more energy-saving. The existing self-balancing bicycles usually adopt the narrow structure of two wheels arranged back and forth, and the wheelbase between their two wheels has an important impact on their balance performance. If the wheelbase increased for improving the load-carrying capacity, it may cause many unexpected problems aroused from the frame deformation and flexibility reduction, and it is easy to lead the bicycle to lose balance.

How to improve the payload capacity without reducing the balance performance of the system? Inspired by the multi-section train, we proposed a new self-balancing bicycle mechanism composed of two-wheel tractor and single-wheel trailer. This kind of two-section bicycle retains the advantages of the traditional bicycle body in structure. If a breakthrough can be made in balance theory and experiment, this kind of mechanism should become a new type of convenient road traffic tool.

The structure of a new tractor-trailer-bicycle (TTB) are described in details in this article, and its dynamic model of the system was developed seriously by using Chaplygin equation.

\section{Mechanical structure}

The TTB consists of roughly two parts: a tractor and a trailer, which is shown in Fig. 1 Fig. 2.

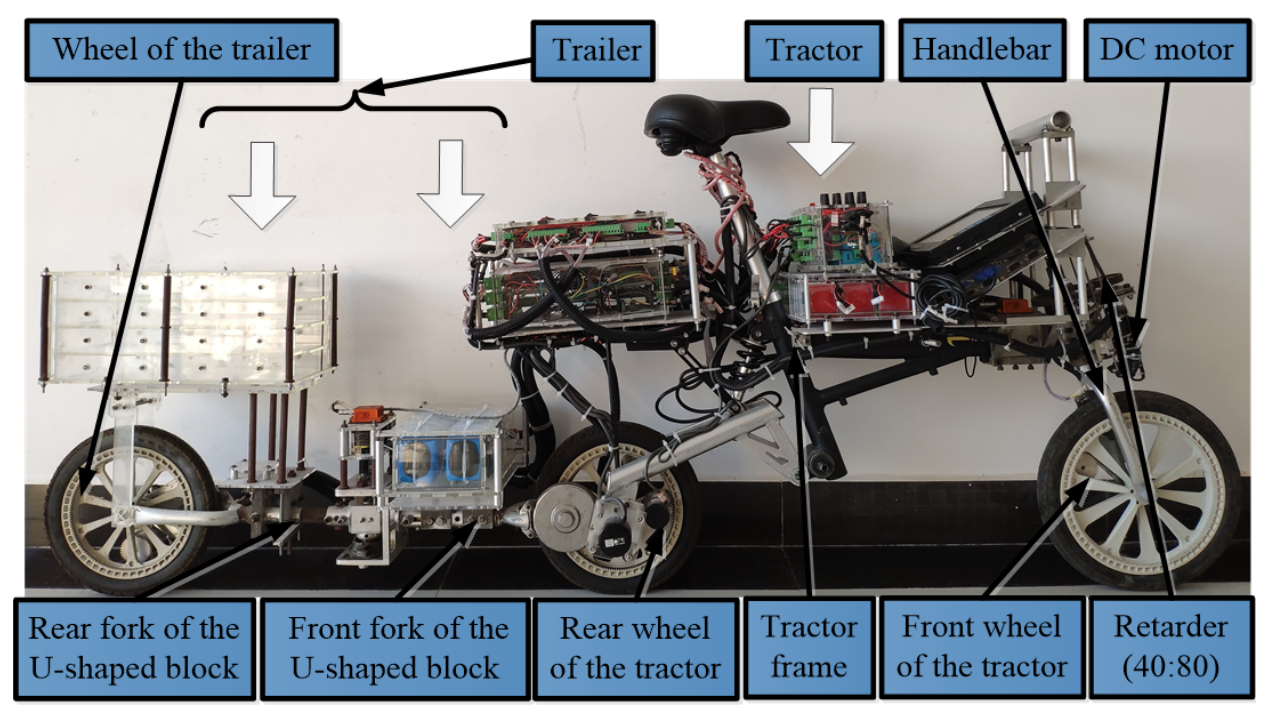

Fig. 1. Physical prototype of our TTB. 


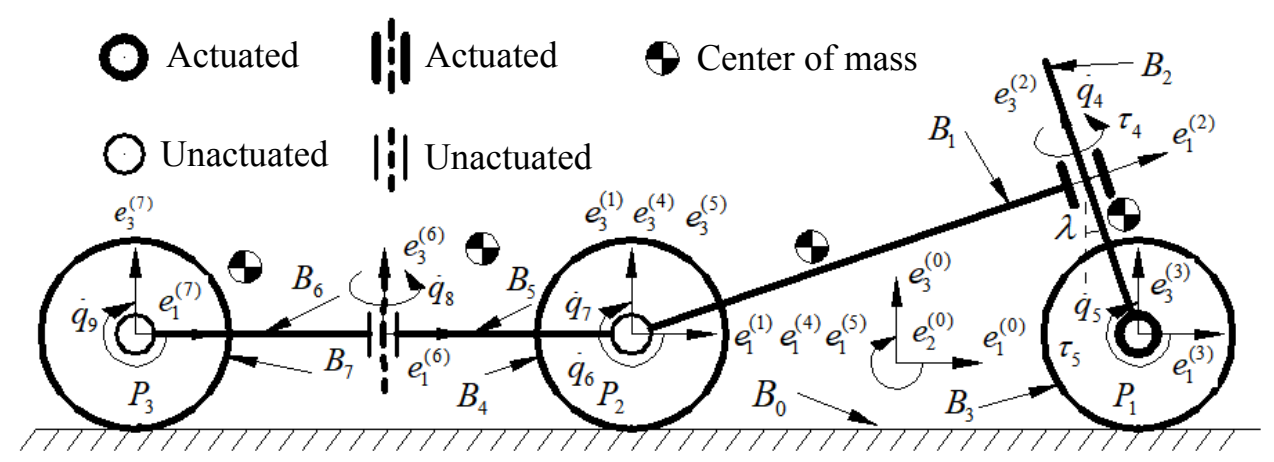

Fig. 2. Schematic diagram of our TTB.

As it is seen in Fig. 1 Fig. 2, the tractor includes a tractor frame, a handlebar, a front wheel of the tractor and a rear wheel. Both the handlebar and the rear wheel of the tractor are rotated around the frame, and the front wheel of the tractor rotates around the handlebar. The trailer comprises the front fork of the U-shaped block, the rear fork of the U-shaped block and the wheel. The front fork of the U-shaped block can be rotated up and down around the tractor frame, the rear fork of the U-shaped block can be rotated around the front fork of the U-shaped block, and the wheel of the trailer is rotated around the rear fork of the U-shaped block.

\section{Dynamical model}

\subsection{Coordination settings}

We denote the tractor frame by $B_{1}$, the handlebar by $B_{2}$, the front wheel of the tractor by $B_{3}$, the rear wheel of the tractor by $B_{4}$, the front fork of the U-block by $B_{5}$, the rear fork of the U-block by $B_{6}$, and the wheel of the trailer by $B_{7}$. The grounding point is denoted by $P_{1}, P_{2}$, and $P_{3}$. The coordinates of the Self-Balancing Tractor-Trailer-Bicycle are set to:

$O-\boldsymbol{e}_{1}^{(0)} \boldsymbol{e}_{2}^{(0)} \boldsymbol{e}_{3}^{(0)}\{0\}$ is the global coordinate system fixed on the ground;

- $O_{1}-\boldsymbol{e}_{1}^{(1)} \boldsymbol{e}_{2}^{(1)} \boldsymbol{e}_{3}^{(1)}\{1\}$ is the coordinate system of the tractor frame $B_{1}$ and the origin of the coordinates is at the geometric center of the front wheel of the tractor $B_{3}$;

- $O_{2}-\boldsymbol{e}_{1}^{(2)} \boldsymbol{e}_{2}^{(2)} \boldsymbol{e}_{3}^{(2)}\{2\}$ is the coordinate system of the handlebar $B_{2}$ and the origin of the coordinates is at the intersection of the frame of the tractor $B_{1}$ and the axis of the handlebar $B_{2}$;

- $O_{3}-\boldsymbol{e}_{1}^{(3)} \boldsymbol{e}_{2}^{(3)} \boldsymbol{e}_{3}^{(3)}\{3\}$ is the coordinate system of the front wheel of the tractor $B_{3}$ and the origin of the coordinates is at the geometric center of the front wheel of the tractor $B_{3}$;

- $O_{4}-\boldsymbol{e}_{1}^{(4)} \boldsymbol{e}_{2}^{(4)} \boldsymbol{e}_{3}^{(4)}\{4\}$ is the coordinate system of the rear wheel of the tractor $B_{4}$ and the origin of the coordinates is at the geometric center of the rear wheel of the tractor $B_{4}$;

- $O_{5}-\boldsymbol{e}_{1}^{(5)} \boldsymbol{e}_{2}^{(5)} \boldsymbol{e}_{3}^{(5)}\{5\}$ is the coordinate system of the front fork of the U-shaped 
block $B_{5}$ and the origin of the coordinates is at the geometric center of the rear wheel of the tractor $B_{4}$;

- $O_{6}-\boldsymbol{e}_{1}^{(6)} \boldsymbol{e}_{2}^{(6)} \boldsymbol{e}_{3}^{(6)}\{6\}$ is the coordinate system of the rear fork of the U-shaped block $B_{6}$ and the coordinate origin is at the intersection of the rear fork of the $\mathrm{U}$-shaped block $B_{6}$ axis and the rotating shaft of the rear fork of the U-shaped block $B_{6}$ around the front fork of the U-shaped block $B_{5}$;

- $O_{7}-\boldsymbol{e}_{1}^{(7)} \boldsymbol{e}_{2}^{(7)} \boldsymbol{e}_{3}^{(7)}\{7\}$ is the coordinate system of the wheel of the trailer $B_{7}$ and the origin of the coordinates is at the geometric center of the wheel of the trailer $B_{7}$.

\subsection{Constraint analysis}

We suppose the bicycle was running on a flat plane, then the angular velocity of $B_{1}$ can be given as:

$$
\boldsymbol{\omega}_{B 1}^{(1)}=\left(c_{3} \dot{q}_{2}-c_{2} s_{3} \dot{q}_{1}\right) \boldsymbol{e}_{1}^{(1)}+\left(s_{2} \dot{q}_{1}+\dot{q}_{3}\right) \boldsymbol{e}_{2}^{(1)}+\left(c_{2} c_{3} \dot{q}_{1}+s_{3} \dot{q}_{2}\right) \boldsymbol{e}_{3}^{(1)}
$$

where $\boldsymbol{e}_{i}^{(j)} \quad(i=1,2,3, j=1,2, \cdots) \quad$ is the $i$ th base vector of coordinate $\{j\} . s_{i}=\sin \left(q_{i}\right), c_{i}=\cos \left(q_{i}\right)(i=1,2, \cdots) ; \dot{q}_{i}(i=1,2,3)$ is the $i$ th Euler angular rate of $B_{1}$.

Because $B_{i}(i=2,4,5)$ rotates about $B_{1}$, their angular velocity should be calculated as:

$$
\boldsymbol{\omega}_{B 2}^{(2)}={ }^{2} \boldsymbol{R}_{1} \cdot \boldsymbol{\omega}_{B 1}^{(1)}+\dot{q}_{4} e_{3}^{(2)}, \quad \boldsymbol{\omega}_{B i}^{(1)}=\boldsymbol{\omega}_{B 1}^{(1)}+\dot{q}_{(i+2)} e_{2}^{(1)}(i=4,5)
$$

where ${ }^{j} \boldsymbol{R}_{i}(i, j=1,2, \cdots)$ denotes the rotation transform matric from the coordinate $\{j\}$ to $\{i\}$, and $\dot{q}_{i}(i=6,7)$ denotes the angular rate of $B_{i}(i=4,5)$, respectively.

Because $B_{3}$ rotates around $B_{2}, B_{6}$ rotates around $B_{5}$, and $B_{7}$ rotates around $B_{6}$, so the expressions of their angular velocity are as follows:

$$
\boldsymbol{\omega}_{B 3}^{(2)}=\boldsymbol{\omega}_{B 2}^{(2)}+\dot{q}_{5} e_{2}^{(2)}, \quad \boldsymbol{\omega}_{B 6}^{(6)}={ }^{6} \boldsymbol{R}_{1} \bullet \boldsymbol{\omega}_{B 5}^{(1)}+\dot{q}_{8} e_{3}^{(6)}, \quad \boldsymbol{\omega}_{B 7}^{(6)}=\boldsymbol{\omega}_{B 2}^{(2)}+\dot{q}_{9} e_{2}^{(6)}
$$

where $\dot{q}_{i}(i=4,5,8,9)$ denotes the angular rate of $B_{i}(i=2,3,6,7)$, respectively.

Assume that the bicycle would not slide on the horizontal plane, so the speed of $P_{1}$ and $P_{2}$ is zero, then there we can get the following equation:

$$
\boldsymbol{v}_{o 3}^{(2)}+\boldsymbol{\omega}_{B 3}^{(2)} \times\left({ }^{2} \boldsymbol{R}_{3} \bullet r_{1}^{(3)}\right)=0, \quad \boldsymbol{v}_{o 4}^{(1)}+\boldsymbol{\omega}_{B 4}^{(1)} \times\left({ }^{1} \boldsymbol{R}_{4} \cdot r_{2}^{(4)}\right)=0
$$

where $r_{i}^{(j)}(i, j=1,2, \cdots)$ denotes the position vector of $P_{i}$ in coordinate $\{j\}$.

In addition, there are the following equations:

$$
\boldsymbol{v}_{o 3}^{(2)}={ }^{2} \boldsymbol{R}_{1} \cdot \boldsymbol{v}_{o 4}^{(1)}+\boldsymbol{\omega}_{B 1}^{(1)} \times \boldsymbol{l}_{B 3}^{(1)}, \quad \boldsymbol{v}_{o 4}^{(4)}=\dot{x} \boldsymbol{e}_{1}^{(4)}+\dot{y} \boldsymbol{e}_{2}^{(4)}
$$

where $l_{B i}^{(j)}(i=1,2, \cdots, 7 ; j=1,5,6)$ denotes the position vector in $\{j\}$ from the center of $B_{i}$ to 
the origin of $\{j\}, v_{o 4}^{(4)}$ is the linear velocity of the geometric center of the rear wheel of the tractor.

In (1) (5) and (8) (11), we can get the following four nonholonomic constraint equations:

$$
\dot{q}_{k}=t_{i} \dot{q}_{2}+t_{i+1} \dot{q}_{4}+t_{i+2} \dot{q}_{5}(k=1,6), \quad \dot{x}=f_{1} \dot{q}_{2}+f_{2} \dot{q}_{4}+f_{3} \dot{q}_{5}, \quad \dot{y}=f_{4} \dot{q}_{2}
$$

Similarly, the speed of $P_{3}$ is zero, so we can get:

$$
\dot{q}_{k}=v_{i} \dot{q}_{2}+v_{i+1} \dot{q}_{4}+v_{i+2} \dot{q}_{5}(k=8,9)
$$

In (12) (15), $\dot{x}, \dot{y}$ denote the longitudinal and the lateral velocity of the geometric center of $B_{4}$, respectively; and $t_{i}, v_{i}, f_{j}(i=1,4 ; j=1,2,3,4)$ is the function of $q_{m}(m=2,3,4,7,8)$.

In addition, considering the geometric constraints, we can get the following equations:

$$
\left\{\begin{array}{l}
\left({ }^{0} \boldsymbol{R}_{1} \cdot \boldsymbol{R}_{2} \cdot{ }^{2} \boldsymbol{R}_{3} \cdot\left(0,0, r_{1}\right)\right)[3]=\left(\left(x_{C 0}, y_{C 0}, r_{1} c_{2}\right)+{ }^{0} \boldsymbol{R}_{1} \cdot \boldsymbol{l}_{B 3}^{(1)}\right)[3] \\
\left({ }^{0} \boldsymbol{R}_{1} \cdot \boldsymbol{R}_{5} \cdot{ }^{5} \boldsymbol{R}_{6} \cdot{ }^{6} \boldsymbol{R}_{7} \cdot\left(0,0, r_{1}\right)\right)[3]=\left(\left(x_{C 0}, y_{C 0}, r_{1} c_{2}\right)+{ }^{0} \boldsymbol{R}_{1} \cdot \boldsymbol{R}_{5} \cdot \boldsymbol{l}_{o 6}^{(5)}+{ }^{0} \boldsymbol{R}_{1} \cdot{ }^{1} \boldsymbol{R}_{5} \cdot{ }^{5} \boldsymbol{R}_{6} \cdot \boldsymbol{l}_{B 7}^{(6)}\right)[3]
\end{array}\right.
$$

Eventually, we can derive the following two holonomic constraint equations:

$$
\dot{q}_{3}=w_{1} \dot{q}_{2}+w_{2} \dot{q}_{4}, \quad \dot{q}_{7}=w w_{1} \dot{q}_{2}+w w_{2} \dot{q}_{4}+w w_{3} \dot{q}_{5}
$$

where $r_{1}$ denote the radius of three wheels, $\left(x_{C 0}, y_{C 0}, r_{1} c_{2}\right)$ denote the position vector of the center of the rear wheel of the tractor in coordinate $\{0\},(*)[3]$ is the 3rd item of the vector*, and $w_{i} 、 w w_{j} \quad(i=1,2 ; j=1,2,3)$ is the function of $q_{k}(k=2,3,4,7,8)$.

We assume that the bicycle is running on a flat ground. The attitude matrix of the front fork of the U-shaped block is denoted by $\boldsymbol{R}_{\alpha}$, and the roll angle is denoted by $\alpha$, and the attitude matrix of the rear fork of the U-shaped block is denoted by $\boldsymbol{R}_{\beta}$, and the roll angle is denoted by $\beta$. As a result, we would get two equations $: \boldsymbol{R}_{a}={ }^{0} \boldsymbol{R}_{1} \cdot{ }^{1} \boldsymbol{R}_{5}$, $\boldsymbol{R}_{\beta}={ }^{0} \boldsymbol{R}_{1} \cdot{ }^{1} \boldsymbol{R}_{5} \cdot{ }^{5} \boldsymbol{R}_{6}$. Therefore, we further have:

$$
\alpha=q_{2}, \quad s_{\beta}=c_{8} s_{2}+c_{2} s_{8} s_{3+7}
$$

From Eq. 16 and Eq. 18 Eq. 21, we can know $\alpha, \beta$ is the function of $q_{i}(i=2,4,5)$.

\subsection{Velocities of the COM}

We set the velocity of the geometric center of $B_{4}$ as:

$$
\boldsymbol{v}_{C 4}^{(4)}=\dot{x} \boldsymbol{e}_{1}^{(4)}+\dot{y} \boldsymbol{e}_{2}^{(4)}
$$

Considering the principle of the relative motion, we can get the velocity of $B_{k}(k=1,2,3,5,6,7)$, respectively, as follows: 


$$
\begin{array}{r}
\boldsymbol{v}_{C i}^{(1)}={ }^{1} \boldsymbol{R}_{4} \cdot \boldsymbol{v}_{C 4}^{(4)}+\boldsymbol{\omega}_{B 1}^{(1)} \times \boldsymbol{l}_{B i}^{(1)}(i=1,2,3), \boldsymbol{v}_{C 5}^{(1)}={ }^{1} \boldsymbol{R}_{4} \cdot \boldsymbol{v}_{C 4}^{(4)}+\boldsymbol{\omega}_{B 5}^{(1)} \times\left({ }^{1} \boldsymbol{R}_{5} \cdot \boldsymbol{l}_{B 5}^{(5)}\right) \\
\boldsymbol{v}_{C j}^{(1)}={ }^{1} \boldsymbol{R}_{4} \cdot \boldsymbol{v}_{C 4}^{(4)}+\boldsymbol{\omega}_{B 5}^{(1)} \times\left({ }^{1} \boldsymbol{R}_{5} \cdot \boldsymbol{l}_{o 6}^{(5)}\right)+{ }^{1} \boldsymbol{R}_{5} \cdot\left(\boldsymbol{\omega}_{B 6}^{(6)} \times \boldsymbol{l}_{B j}^{(6)}\right)(j=6,7)
\end{array}
$$

where $\boldsymbol{v}_{C i}^{(1)}$ is the velocity of $B_{i}(i=1,2,3,6,7)$ in $\{1\}, \quad \boldsymbol{I}_{o 6}^{(5)}$ is the position vector in $\{5\}$ from the origin of $\{6\}$ to the origin of $\{5\}$.

\subsection{Kinetic energy and potential energy}

According to the derived $\boldsymbol{\omega}_{B i}^{(j)}(i, j=1,2, \cdots, 5)$ in Eq. $1 \sim$ Eq. 7 and $\boldsymbol{v}_{C i}^{(k)}(i=1,2, \cdots, 7 ; k=1,4)$ in Eq. 22 Eq. 28, we can calculate the system's kinetic energy as:

$$
T=\frac{1}{2} \sum_{i=1}^{5}\left(\left(\boldsymbol{\omega}_{B i}^{(j)}\right)^{T} \boldsymbol{J}_{B i}\left(\boldsymbol{\omega}_{B i}^{(j)}\right)+\left(\boldsymbol{v}_{C i}^{(k)}\right)^{T} M_{B i}\left(\boldsymbol{v}_{C i}^{(k)}\right)\right)
$$

where $\boldsymbol{J}_{B i}\left(\boldsymbol{M}_{B i}\right)(i=1,2, \cdots, 7$ )represents the inertial matric(mass matrix)of $B_{i}(i=1,2, \cdots, 7)$, respectively.

By substituting the nonholonomic constrains Eq. 12 Eq. 17 into $T$, we will get another form of the kinetic energy $\tilde{T}$. Simultaneously, system gravity potential $U$ can be given as:

$$
U=\sum_{i=1}^{7} m_{i} g h_{i}(i=1,2, \cdots, 7)
$$

where $m_{i}$ and $h_{i}$ are the mass and center height(the ground plane as the zero potential energy surface) of $B_{i}(i=1 \sim 7)$, respectively. The formula of the height of each rigid body is as follows:

$$
\begin{array}{r}
h_{4}=r_{1} c_{2}, \quad h_{5}=h_{4}+\left({ }^{0} R_{1} \cdot{ }^{1} R_{5} \cdot l_{C_{5}}^{(5)}\right)[3], \quad h_{j}=h_{4}+\left({ }^{0} R_{1} \bullet l_{C_{j}}^{(1)}\right)[3](j=1,2,3) \\
h_{k}=h_{4}+\left({ }^{0} R_{1} \bullet R_{5} \cdot{ }^{5} R_{6} l_{C_{6}}^{(6)}\right)[3]+\left({ }^{0} R_{1} \bullet R_{5} \cdot l_{o_{k}}^{(5)}\right)[3](k=6,7)
\end{array}
$$

\subsection{Dynamical model}

Considering the following form of Chaplygin equation:

$$
\frac{d}{d t} \frac{\partial \tilde{T}}{\partial \dot{q}_{\sigma}}-\frac{\partial \tilde{T}}{\partial q_{\sigma}}-\sum_{\beta=1}^{\gamma} \frac{\partial T}{\partial \dot{q}_{\varepsilon+\beta}} \sum_{v=1}^{\varepsilon}\left(\frac{\partial B_{\varepsilon+\beta, \sigma}}{\partial q_{v}}-\frac{\partial B_{\varepsilon+\beta, v}}{\partial q_{\sigma}}\right) \dot{q}_{v}=\tilde{Q}_{\sigma}
$$

where $T$ is the kinetic energy and $\tilde{T}$ is the kinetic energy by substituting nonholonomic constrains into $T ; B_{\varepsilon+\beta, \sigma}$ is the $\sigma$ th coefficient of the $\beta$ th nonholonomic constrain; $q_{\nu}$ and $q_{\sigma}$ are the generalized coordinates of the system; $\varepsilon$ and $\gamma$ are the numbers of the independent generalized coordinates and the nonholonomic constrains; $\tilde{Q}_{\sigma}$ is the $\sigma$ th generalized force of the system. The system's dynamics as: 


$$
\boldsymbol{D}(\boldsymbol{q}) \ddot{\boldsymbol{q}}_{\theta}+\boldsymbol{C}\left(\boldsymbol{q}, \dot{\boldsymbol{q}}_{\theta}\right) \dot{\boldsymbol{q}}_{\theta}+\boldsymbol{G}(\boldsymbol{q})=\boldsymbol{\tau}
$$

In (39), $\boldsymbol{C}\left(\boldsymbol{q}, \dot{\boldsymbol{q}}_{\theta}\right)=\left(C_{i j}\right)_{3 \times 3}, \boldsymbol{G}(\boldsymbol{q})=\left(\begin{array}{lll}G_{1} & G_{2} & 0\end{array}\right)^{T}$, and $\boldsymbol{D}(\boldsymbol{q})=\left(D_{i j}\right)_{3 \times 3}$ denote centripetal-Coriolis, gravity terms and the inertia; $\boldsymbol{\tau}=\left(0, \tau_{4}, \tau_{5}\right)^{T}$ denote the driving torque vector; $\boldsymbol{q}_{\theta}$ and $\boldsymbol{q}$ are two kinds of generalized coordinates, which are defined as: $\boldsymbol{q}_{\theta}=\left(q_{2}, q_{4}, q_{5}\right)^{T}, \boldsymbol{q}=\left(q_{1}, q_{3}, q_{7}, q_{8}\right)^{T}$.

Eq. 39 indicates the TTB is an under-actuated system with three independent velocities, and for more detail: The roll angle $\left(q_{2}, \alpha, \beta\right)$ of $B_{1}, B_{5}, B_{6}$ are under-actuated,and $\alpha, \beta$ is the function of $q_{i}(i=2,4,5)$; There are totally two driving inputs in the two joints $q_{i}(i=4,5)$, so we could regulate control-force inputs $\tau_{i}(i=4,5)$ of the two joints to control the roll angle $\left(q_{2}, \alpha, \beta\right)$ of the TTB.

\section{Model verification}

We will demonstrate the reliability of the model (see Eq. 39) by two different approaches. One is the use of an inverse dynamic simulation of Eq.39 under a given balanced trajectory in Matlab, from which we compare the energy increment with the input work of the dynamic bicycle. The other is the use of a virtual prototype simulation in ADAMS, by which we compare the driving torque of the handlebar in Adams with the model-calculated handlebar driving torque.

Table 1 shows the physical parameters which would be used in the numerical simulation. Note that we obtain the parameters from the measurement of a virtual TTB prototype in Solidworks.

Table 1. Physical parameters.

\begin{tabular}{|c|c|c|c|c|c|c|c|c|c|c|}
\hline \multirow{2}{*}{$\frac{\text { Symbol }}{l_{1}}$} & \multirow{2}{*}{$\frac{\text { Value }}{0.250}$} & \multirow{2}{*}{$\frac{\text { Unit }}{\mathrm{m}}$} & \multirow{2}{*}{$\frac{\text { Symbol }}{l_{B 7}^{(6)}}$} & \multicolumn{3}{|c|}{ Value } & \multirow{2}{*}{$\frac{\text { Unit }}{\mathrm{m}}$} & \multirow{2}{*}{$\frac{\text { Symbol }}{m_{1}}$} & \multirow{2}{*}{$\frac{\text { Value }}{25.038}$} & \multirow{2}{*}{$\frac{\text { Unit }}{\mathrm{kg}}$} \\
\hline & & & & $(-0$. & 347 & 0) & & & & \\
\hline & \multirow{4}{*}{$(0.275,0,0.145)$} & & & (3.106 & 0 & 0 & & & & \\
\hline \multirow[t]{3}{*}{$l_{B 1}^{(1)}$} & & \multirow[t]{3}{*}{$\mathrm{m}$} & \multirow[t]{3}{*}{$J_{B 1}$} & 0 & 0.581 & 0 & \multirow[t]{3}{*}{$\mathrm{kg} \cdot \mathrm{m} 2$} & \multirow[t]{3}{*}{$m_{2}$} & \multirow[t]{3}{*}{2.946} & \multirow[t]{3}{*}{$\mathrm{kg}$} \\
\hline & & & & 0 & 0 & $2.629)$ & & & & \\
\hline & & & & $(0.070$ & 0 & 0 & & & & \\
\hline \multirow[t]{3}{*}{$l_{B 2}^{(1)}$} & \multirow[t]{3}{*}{$(0.070,0,0.166)$} & \multirow[t]{3}{*}{$\mathrm{m}$} & \multirow[t]{3}{*}{$J_{B 2}$} & 0 & 0.073 & 0 & \multirow[t]{3}{*}{$\mathrm{kg} \cdot \mathrm{m} 2$} & \multirow[t]{3}{*}{$m_{w}$} & \multirow[t]{3}{*}{1.616} & \multirow[t]{3}{*}{$\mathrm{kg}$} \\
\hline & & & & 0 & 0 & $0.008)$ & & & & \\
\hline & & & & $(1.000$ & 0 & 0 & & & & \\
\hline \multirow{3}{*}{$l_{B 3}^{(1)}$} & \multirow[t]{3}{*}{$(0.893,0,0)$} & \multirow[t]{3}{*}{$\mathrm{m}$} & \multirow[t]{3}{*}{$J_{B 3}$} & 0 & 0.500 & 0 & \multirow[t]{3}{*}{$\mathrm{kg} \cdot \mathrm{m} 2$} & \multirow[t]{3}{*}{$m_{5}$} & \multirow[t]{3}{*}{2.294} & \multirow[t]{3}{*}{$\mathrm{kg}$} \\
\hline & & & & 0 & 0 & $1.000)$ & & & & \\
\hline & & & & $(0.040$ & 0 & 0 & & & & \\
\hline \multirow[t]{3}{*}{$l_{B 5}^{(1)}$} & \multirow[t]{3}{*}{$(-0.274,0,0.036)$} & $\mathrm{m}$ & $J_{B 5}$ & 0 & 0.070 & 0 & $\mathrm{~kg} \cdot \mathrm{m} 2$ & $m_{6}$ & 2.070 & $\mathrm{~kg}$ \\
\hline & & & & 0 & 0 & $0.040)$ & & & & \\
\hline & & & & 0.033 & 0 & 0 & & & & \\
\hline$l_{B 6}^{(6)}$ & $(-0.204,0,0.102)$ & $\mathrm{m}$ & $J_{B 6}$ & 0 & 0.056 & 0 & $\mathrm{~kg} \cdot \mathrm{m} 2$ & $r_{1}$ & 0.200 & $\mathrm{~m}$ \\
\hline & & & & 0 & 0 & 0.060 & & & & \\
\hline
\end{tabular}




\subsection{Inverse dynamics simulations}

The simulation is perform with two steps.

\section{Step 1: trajectory planning}

We plan the motion trajectory of the roll angle of the tractor frame $B_{1}$ and the angular velocity of the front wheel of the tractor $B_{5}$ :

$$
q_{2}=A_{1} e^{-\sigma t} \sin (\omega t+\varphi), \quad \dot{q}_{5}=A_{2}, \quad \ddot{q}_{5}=0 .
$$

where $A_{1}=\pi / 35, A_{2}=6 \pi, \sigma=-1, \omega=\pi, \varphi=\pi / 2$.

\section{Step 2: : driving torque calculating}

By solving Eq. 39, we can calculate the driving torque $\tau_{4}$ of the handlebar $B_{2}$ and the driving torque $\tau_{5}$ of the front wheel of the tractor $B_{5}$, and then we will obtain:

$$
\tau_{4}=\left(D_{21}-\frac{D_{11} D_{22}}{D_{12}}\right) \ddot{q}_{2}-\frac{D_{11} F_{1}}{D_{12}}+F_{2}, \quad \tau_{5}=\left(D_{31}-\frac{D_{11} D_{32}}{D_{12}}\right) \ddot{q}_{2}-\frac{D_{32} F_{3}}{D_{12}}+F_{2}
$$

Here, $F_{1}=C_{11} \dot{q}_{2}+C_{12} \dot{q}_{4}+G_{1}, F_{2}=C_{21} \dot{q}_{2}+C_{22} \dot{q}_{4}+G_{2}, F_{3}=C_{31} \dot{q}_{2}+C_{32} \dot{q}_{4}+G_{3}$.

Fig. 3 examines two kinds of kinetic energy: T1 is get by the current velocity and $\mathrm{T} 2$ is get by the previous velocity and the elementary work.

Fig. 4 shows the difference between the mechanical energy and the work of the running bicycle.

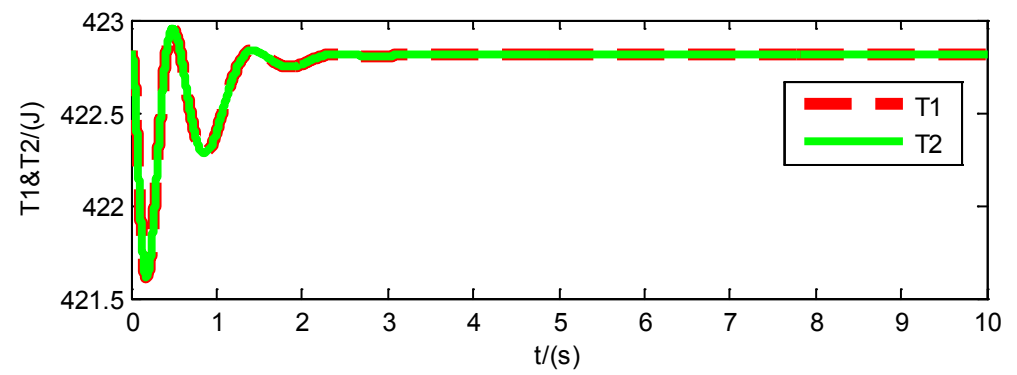

Fig. 3. Comparison of two kinds of kinetic energy.

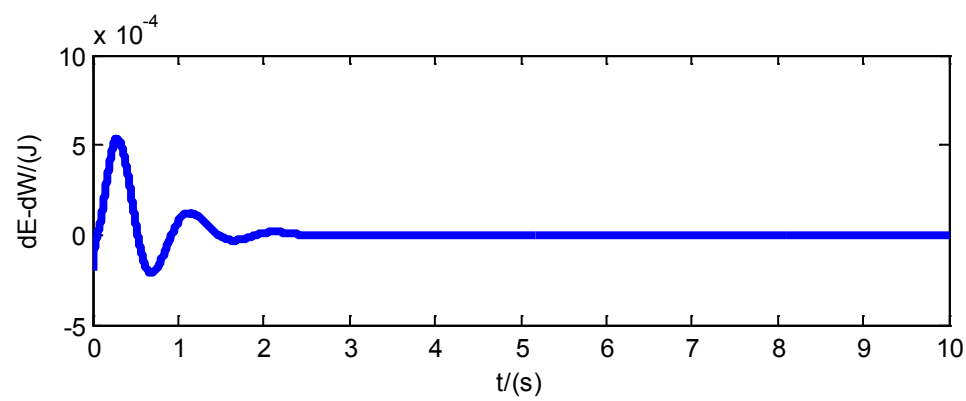

Fig. 4. Difference between energy and work.

As seen in Fig. 3 Fig. 4, while calculated by use of different variable, the two kinetic energy are coincident, and the difference between the increment of the mechanical energy 
and the elementary work is less than $10^{-3}$ (see Fig. 4). The results show that our dynamical model (Eq. 39) strictly obey the law of conservation of energy.

\subsection{Virtual prototype simulation}

The simulation is as follows:

\section{- Experiment description}

First, we build the virtual prototype in Adams platform, and add kinematic pairs and constraints to each rigid body part. Secondly, we define the type of contact between the wheels of the TTB and the ground, and add static friction and dynamic friction. The parameter settings such as system quality, moment of inertia, length and body structure length required for simulation are shown in Table 1.Finally, the angular velocity of the $B_{3}$ is set to $650 \mathrm{r} / \mathrm{min}$, then a simply PD controller is designed as:

$\tau_{4}=k_{p 1} q_{2}+k_{d 1} \dot{q}_{2}$, in which $k_{p 1}=15, k_{d 1}=5$.

The handlebar is governed by the controller to balance the TTB. The simulation continue with 20 s due to the space limit. The relative data of the virtual prototype are exported for the post process after the complement of the simulation.

\section{- Experiments result and analysis}

We calculated the driving torque of the handlebar $B_{2}$ through the dynamical model in Eq. 39. Also, we got the measurement of this torque from ADAMS.

Fig. 5 show that TTB is running on a flat plane in the ADAMS simulation platform environment.

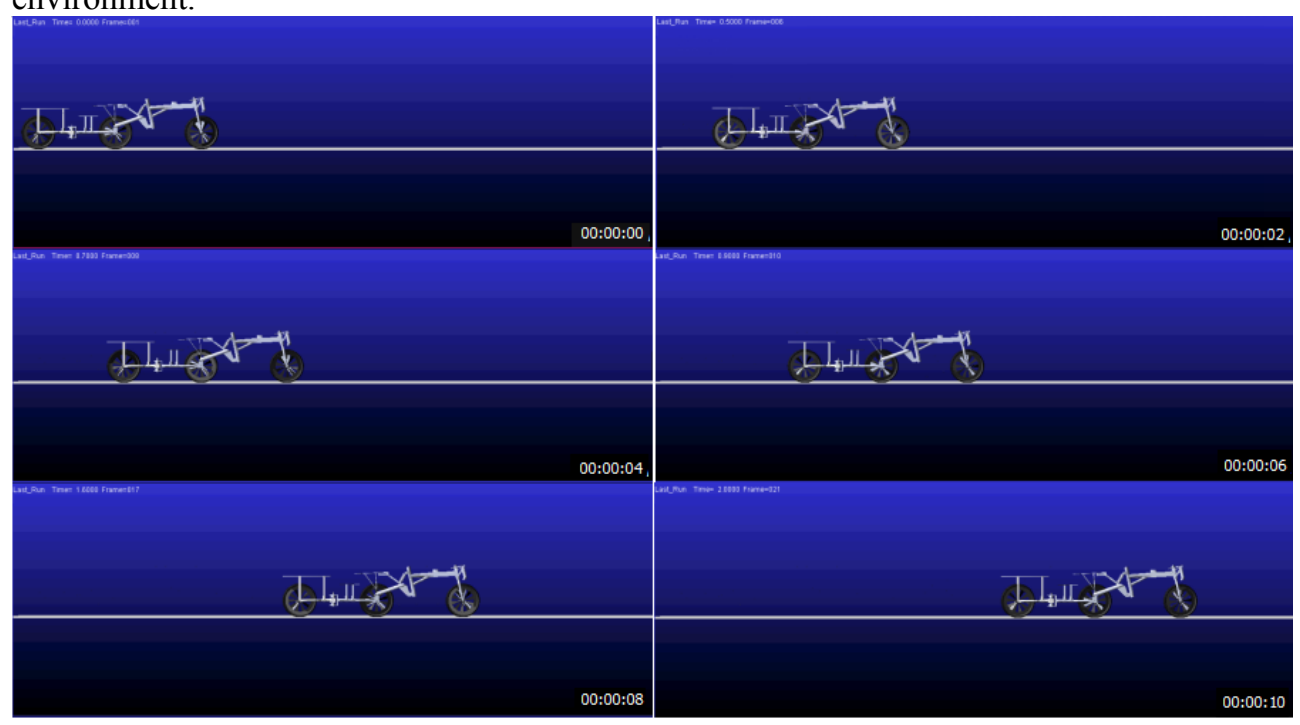

Fig. 5. Snapshot of the balanced running TTB in ADAMS.

Fig. 6 show the results of the analysis. 


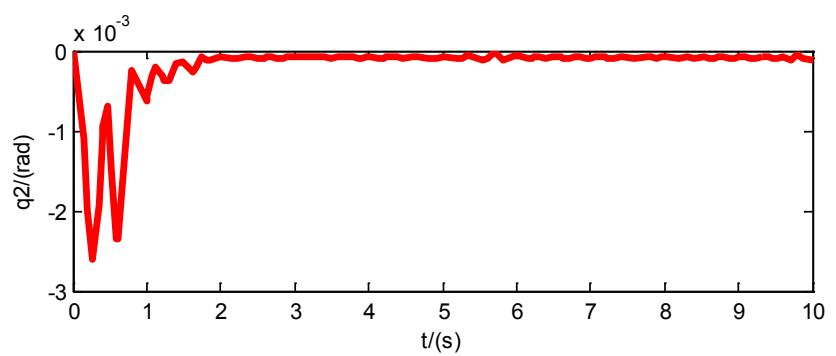

a) the roll angle of the tractor frame

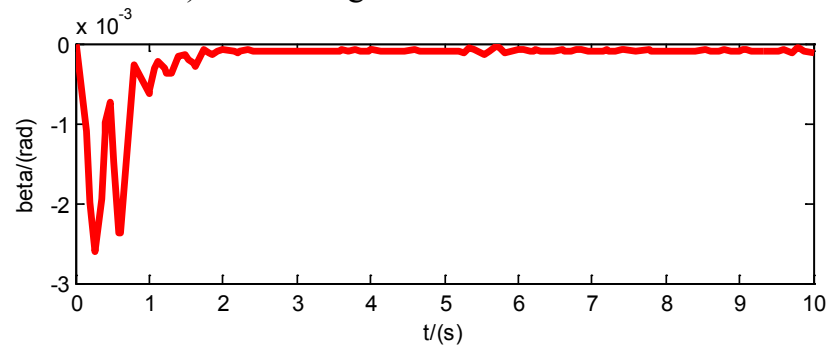

b) the roll angle of the rear fork of the U-block

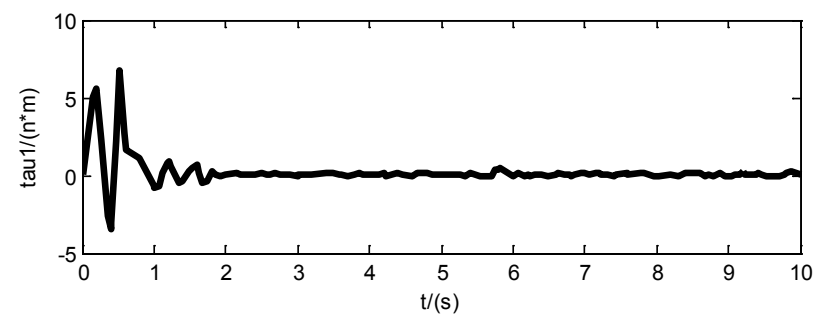

c) the angular acceleration of the front wheel of the tractor

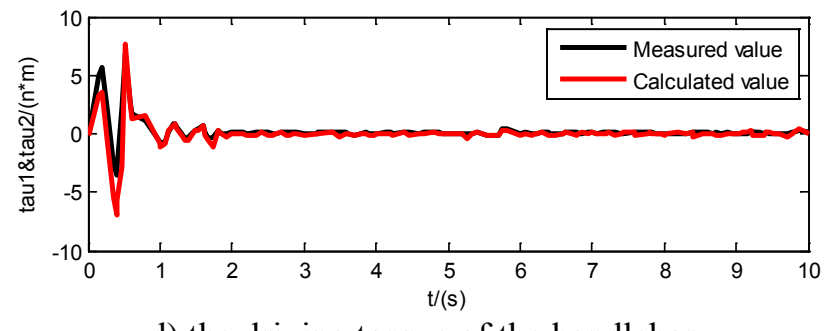

d) the driving torque of the handlebar

Fig. 6. The data to be exported in Adams.

It is illustrated that the two kinds of the driving torque of the handlebar $B_{2}$ exhibited the similar trend with a little difference in the amplitude. The reasons for the difference maybe as follows:

1) There definitely is wheel slippage because the angular acceleration of the front wheel of the tractor $B_{5}$ is relatively large form 0 to 0.5 second; 2) The measurement errors of virtual sensors; 3) The structural parameter measurement errors; 4) The proposed dynamical model is developed under ideal assumption without considering the friction between the wheels and the ground etc.

As a conclusion, the results of the two simulations seriously demonstrate the correctness and the reliability of the proposed dynamical model (Eq. 39). 


\section{Conclusions and future work}

One of the contribution of this research is that we suggest that the TTB can be controlled balance by the handlebar. Another contribution of the research might be that we explored the dynamical model for the system. Our model illustrates that the TTB is explicitly a nonholonomic and under-actuated system, which consist of three independent velocities and two control-torque inputs. With the comparison between numerical and virtual prototype simulation, we validated that the reliability of our dynamical model. However, by so far, there is lack of realistic test to provide further support for our theoretical analysis; so, our next work should concentrate on the physical experiments.

Our work has received the financial support from National Natural Science Foundation of China (Grant No. $51765011 \& \&$ No. 51865005) and Project of Guangxi Natural Science Foundation (Grant No. 2018JJA160115 \&\& No. 2018JJA160116).

\section{References}

1. Jones D E H. The stability of the bicycle[J]. Physics Today, 1970, 23(4): 34-40.

2. Tanaka Y, Murakami T. Self sustaining bicycle robot with steering controller[C]// The 8th IEEE International Workshop on Advanced Motion Control, Kawasaki International Center, Kawasaki, Japan. Kawasaki: IEEE, 2004: 193-197.

3. Kooijman J D G, Meijaard J P, Papadopoulos J M. A bicycle can be self-stable without gyroscopic or caster effects [J]. Science Magazine, 2011, 332(6027): 339-342.

4. Huang Y H, Liao Q Z, Guo L, et al. Simple realization of balanced motions under different speeds for a mechanical regulator-free bicycle robot[J]. Robotica, 2014, 72(9): $1-15$.

5. Huang Y H, Liao Q Z, Guo L, et al. Balanced motions realization for a mechanical regulators free and front-wheel drive bicycle robot under zero forward speed[J]. International Journal of Advanced Robotic Systems, 2013, 10(317):1-9.

6. Li J, Wei S M, Guo L, et al. Adaptive fuzzy control of a front-wheel drive bicycle $\operatorname{robot}[\mathrm{C}] / / 2016$ 4th International Conference on Cloud Computing and Intelligence Systems, IEEE, 2016: 113-116.

7. Lee S, Ham W. Self stabilizing strategy in tracking control of unmanned electric bicycle with mass balance[C]// Proceedings of the 2002 IEEE/RSJ International Conference on Intelligent Robots and Systems, Lausanne, Switzerland. Lausanne: IEEE, 2002: 2200-2205.

8. Bui T T, Parnichkun M, Le C H. Structure-specified Hळ loop shaping control for balancing of bicycle robots: A particle swarm optimization approach[J]. Proceedings of the Institution of Mechanical Engineers. Part I: Journal of Systems and Control Engineering, 2010, 224(7): 857-867.

9. Liu Y B, Jia C H, Han J H. Dynamics modeling of an unmanned bicycle with parallel mechanism adjusting stability[C]// Proceedings of the 2009 IEEE International Conference on Mechatronics and Automation, Changchun, China. Changchun: IEEE, 2009: 1601-1605.

10. Jin H Z, Yang D C, Liu Z X, et al. A gyroscope-based inverted pendulum with application to posture stabilization of bicycle vehicle[C]// Proceedings of the 2015 IEEE Conference on Robotics and Biomimetics, Zhuhai, China. Zhuhai: IEEE, 2015:2103-2108. 
11. Yin S, Yamakita M. Passive velocity field control approach to bicycle robot path following [C]// Proceedings of the SICE Annual Conference, Tsukuba, Japan. Tsukuba : IEEE, 2016: 1654-1659.

12. Kim Y, Kim H, Lee J. Stable control of the bicycle robot on a curved path by using a reaction wheel[J]. Journal of Mechanical Science and Technology, 2015, 29 (5): 2219-2226. 